\title{
GARGINOMA OF THE PROSTATE.
}

\author{
By J. SWIFT JOLY, F.R.C.S. \\ (Senior Surgeon to St. Peter's Hospital for Stone.)
}

Until the end of the last century carcinoma of the prostate was thought to be a comparatively rare condition. It was only in the first decade of this century that its frequency was generally recognised. It is probable that before this period it was confused with the benign senile enlargement of the organ.

The age incidence is comparatively limited. Three-quarters of the patients are between sixty and seventy-five when they come under observation, but as the condition is usually advanced and inoperable when the patient is first seen, the average age of the patient when the carcinoma first arose must have been a year or two less than the figures given above. It is really impossible to state accurately the time of onset, as an early carcinoma gives rise to no symptoms. It is, however, certain that the average age of these patients is at least five years less than that of patients suffering from benign enlargement.

There are two forms of prostatic carcinoma. In the first, the malignant disease arises in prostates that are apparently normal, or at least do not give rise to obstructive symptoms. In the second, there is a malignant degeneration of a hypertrophied gland. The first is the more common of the two, and tends to affect younger patients.

The frequency of the condition is best estimated by comparing it with the frequency of benign enlargement. About 20 per cent. of all patients who seek relief from senile prostatic obstruction suffer from carcinoma. Statistics drawn from different sources naturally vary, but this figure is approximately correct. To this must be added cases in which an unsuspected carcinoma is discovered on microscopic examination of the enucleated gland. Different surgeons give very varying figures for the frequency in which an unsuspected carcinoma is thus found. Albarran puts the proportion at I4 per cent., Young at 20 per cent., ThomsonWalker stated that in 16 per cent. of the prostates removed by enucleation the gland showed either " malignant or premalignant" changes. On the other hand, Blum found malignancy in 6 per cent. of the prostates he removed, Hryntschak found cancerous degeneration in 3 per cent. of over 300 enlarged prostates, and in a further 7 per cent. he found changes " which were looked upon as precancerous." My own figures agree with those of Hryntschak. In 4 per cent. of my cases there was an error of diagnosis. In 3 per cent. a carcinoma was missed, while in the remaining I per cent. chronic inflammatory changes were thought to be malignant. This diversity of opinion shows that different pathologists have different ideas as to what constitutes the first sign of malignancy, and also as to whether certain conditions, such as proliferation of the epithelial cells, should be classed as cancerous or precancerous. Still, the fact remains that taking all forms of cancer of the prostate together, about a quarter of the patients seeking relief from prostatic obstruction suffer from malignant disease.

Etiology. Nothing is known for certain on this point. It has been stated that previous prostatitis, posterior urethritis, stricture, etc., favour the formation of prostatic carcinoma, but I feel that this is not the case as the urine is always sterile, unless the bladder has been infected by catheterization. In addition, stricture and other signs of a urethritis are very rarely present. The carcinoma appears to arise in normal glands. In cases where it arises as a degeneration of benign hypertrophy, there is no known factor that makes this change probable.

\section{Carcinoma arising in an apparently normal gland.}

Symptoms. The early symptoms are very much the same as those of an ordinary benign enlargement. There is a gradually increasing frequency of micturition, which is first noticed at night, but later is diurnal as well. This frequency increases much more rapidly than does the frequency of a benign hypertrophy, and six months after the onset of this symptom, the patient may have to rise four or five times every night. At the same time the urinary stream 
becomes steadily thinner and weaker, and there may be a distinct delay before it starts. Precipitancy of micturition is also a troublesome symptom, but it does not arise until there is a considerable amount of residual urine. It is very unpleasant, as immediately the desire to micturate is experienced, a little urine escapes into the clothing. The frequency and difficulty in micturition steadily increase in severity until finally complete retention sets in. Once this happens, it is exceedingly rare for the patient to be able to pass urine again. On the other hand, a patient with a benign prostate usually recovers the power of spontaneous micturition, and may have several attacks of retention before he finally has to depend on his catheter.

The amount of residual urine increases steadily with the increasing difficulty and frequency. As a rule complete retention occurs when the residual urine reaches 20 to 25 or 30 ounces. For this reason over-distention with overflow incontinence is a comparatively rare symptom. The duration of these symptoms is much shorter when the prostate is malignant than when it is benign. In the former condition they last for about a year or eighteen months before retention occurs, in the latter they usually last for several years. If the patient decides on self-catheterisation he finds that at first he has no difficulty in inserting the instrument, but that it gradually becomes more and more difficult. The result is that he uses smaller and smaller instruments, and finally may be unable to insert any catheter at all. If this happens, it is a good plan to dilate the urethra with steel sounds, as this will for a time relieve the difficulty in instrumentation. The difficulty in passing the catheter is due to a slowly increasing stenosis of the prostatic urethra brought about by the encroachment of the neoplasm on its lumen. At the same time the urethra becomes rigid and inelastic so that there is increasing difficulty in dilating it. When this happens, a suprapubic cystotomy must be performed.

Examination. The diagnosis is usually made by rectal palpation. It is important that the bladder should be emptied before this examination is made, as a full bladder tends to limit the mobility of the gland, and to obscure its outline. The examination is best carried out in the knee-elbow position. In the earliest stages of the disease, the prostate appears to be approximately normal in size. Its surface is smooth and regular, and its outline is clearly defined. One lobe, however, is much harder than the other, and one may obtain the impression that there is a hard central nodule, which is covered by a thin layer of softer tissue. In this stage the whole gland is movable. It is very rare to encounter a carcinoma in such an early stage, as it then does not give rise to symptoms. Later, the gland is uniformly hard, but one lateral lobe is more prominent than the other. The vertical median furrow between the lateral lobes tends to fill up, and becomes obliterated. A certain amount of induration may be felt as a band of firm tissue running upwards and outwards from the upper and outer portion of the margin of the gland. This indurated tissue at first lies just below the seminal vesicle, which may be distended and easily palpable. The mobility of the gland is distinctly impaired, although it is not yet completely fixed. Occasionally a small hard nodule may be felt in the band of induration. It is a small gland, which is usually found in this situation.

In the next stage, the first impression the surgeon obtains is that the gland is greatly enlarged, but a more careful examination soon shows that this is not the case. The carcinoma has spread widely beyond the confines of the prostate, and gives the appearance of a greatly enlarged gland. The spread is most marked upwards and outwards from the upper portions of the lateral border, and the seminal vesicles are completely enveloped in indurated tissue, and are no longer palpable. The space between them is also filled with malignant tissue, which extends upwards to the reflection of the peritoneum at the bottom of the pouch of Douglas. The upper border of this mass of induration may easily be mistaken for the upper border of the prostate. The upper limit of the gland can often be made out as a faintly marked transverse groove a short distance above the middle of the indurated mass. The induration also tends to spread laterally and downwards beyond the capsule of the prostate, but the true limits of the gland can usually be made out. The whole mass is heart-shaped, but the gland only occupies the lower and medial portion. It is extremely hard, absolutely fixed, and has a somewhat irregular and nodular surface.

In the final stage, a large hard mass fills the greater part of the true pelvis. The rectum is displaced backwards into the hollow of the sacrum, and its lumen is encroached upon 
laterally by bands that extend upwards and backwards from the parent mass. The outer coats of the rectum may be involved, but it is exceedingly rare for the carcinoma to ulcerate into the lumen of the bowel. Usually the rectal mucous membrane remains freely movable over the mass. The narrowing of the lumen of the rectum may give rise to a certain amount of constipation, but I have never seen a case where a colostomy was necessary. These large tumour masses can be felt on suprapubic palpation, provided the bladder has been emptied. They appear as rather indefinite masses, usually a short distance from the middle line, or occasionally immediately above the pubis. At the same time the lymph nodes round the external iliac artery may be palpable.

Extensions of the growth into the bladder are not uncommon in the late stages. They are usually found near the lateral margin of the trigone, and are due to an upward extension of the carcinoma from the region of the seminal vesicles through the bladder base. At the same time the tissues at the bladder neck are infiltrated. This renders the internal meatus rigid and inextensible.

Metastases. One of the remarkable features of prostatic carcinoma is its great tendency to form bony metastases. They are sufficiently advanced to be recognised by the naked eye in about 70 per cent. of cases. According to Kaufmann no other carcinoma shows such a marked tendency to form widespread bony metastases. The lumbar spine is most often involved, but metastases have been found in all parts, from the cervical region to the coccyx. The pelvic girdle is next most frequently affected, but it is much more common to find growth in the ilium than in the pubic bone or the ischium. The femur, especially the upper third, is the next most common site. The ribs, sternum and the flat bones of the skull are also frequently involved. It is usually stated that bony metastases are rare below the elbow and knee, but the tibia, the fibula, and the bones of the forearm are not infrequently the site of metastases from prostatic carcinoma. At the same time, if an X-ray examination does not reveal metastases in the lumbar spine, the pelvis and the upper third of the femur, it is exceedingly unlikely that any other bony metastases are present.

In most instances the growth is osteoplast , and the periosteum takes part in it. The result is that the bones are greatly thickened, and appear to be composed entirely, or almost entirely, of irregular spongy tissue. When the bone is macerated, it has a rough porous surface, like a piece of pumice stone. It is irregularly nodular. In extreme cases, the thickening of the bony pelvis may be so marked that the pelvic cavity is greatly reduced in size, the obturator foramen almost obliterated, and the crest of the ilium may be more than two inches in thickness. Similarly, the upper third of the femur may be two or three times thicker than normal, and the trochanters hidden by irregular osteoplastic outgrowths. In other cases, the compact tissue is reduced in amount, so that a spontaneous fracture is prone to occur. It is interesting to note that both osteoplastic and osteoclastic changes are frequently found in different portions of the same bone. Spontaneous fracture is by no means uncommon and usually occurs in the upper third of the femur, but may occur in the humerus or ribs. It is generally transverse.

Less frequently there is very extensive lymphatic involvement. In several instances I have found enlarged nodes in the left supra-clavicular fossa on the first occasion the patient came for treatment. In other cases, enlargement of the aortic nodes has given rise to a large and easily palpable retroperitoneal swelling. It is a curious fact that bone metastases are rare in cases where there is a widespread involvement of the lymph nodes, and conversely, extensive lymph node enlargement is rare when there are obvious bone metastases. It is extraordinary how, in some cases, practically every lymph node in the body becomes infected with cancer cells, while visceral deposits may be absent or only small. At the same time, the pelvic lymph nodes are enlarged in most cases of prostatic carcinoma, and may cause œdema of the leg from pressure on the external or common iliac vein. It is only when the lymphatic involvement is very extensive, that there appears to be an antagonism between the lymphatic and the bony spread of the growth.

Visceral metastases are not common. The liver and the lungs are the organs most commonly involved. 
Symptoms due to metastases. In cases where extensive bone metastases are found, one would expect that they would give rise to pain or other symptoms, but this only occurs in about 30 per cent. of the cases. The pain is either localised to the bone involved, or is referred. The local pain is a constant dull ache, or is often described as a " boring pain." It is often increased by pressure, when the alteration in the thickness or contour of the bone may be noticed. A dull pain felt anywhere in the back should raise suspicion of a secondary deposit in the spinal column at the same level.

The second type of pain is due to pressure on the spinal nerves. As the lower part of the vertebral column is most prone to invasion, the pain is usually referred to the leg. When the deposits are situated in the lumbar region, pain is felt on the anterior aspect of the thigh, or the inner border of the foot. Pain down the posterior aspect of the thigh or leg may be due to pressure on the nerve roots as they pass through the sacral foramina, or to pressure on the great and small sciatic nerves as they emerge from the pelvis. Keyes states that bilateral sciatica in an old man is almost pathognomonic of prostatic cancer. This nerve pain may be a dull ache, or may be paroxysmal and very severe.

Pressure on the veins by enlarged lymph nodes may give rise to marked œdema. After a time the collateral circulation opens up, and the œdema tends to disappear.

Diagnosis. In most instances the diagnosis of carcinoma of the prostate is made by rectal examination, which has already been described. The chief diagnostic points are (I) the hardness of the gland, (2) the absence of the median furrow, (3) the irregularity of the surface, (4) extension of the growth beyond the limits of the gland, and (5) fixation of the whole mass.

A stony hard gland is usually malignant, but may also be due to calculi. Prostatic calculi are usually multiple, and crepitus can often be made out. The diagnosis of stone is confirmed by an X-ray examination. Exceptionally a prostatic carcinoma is soft. This may be due to the fact that the growth itself is of a soft medullary type, or else that it contains pseudo-cysts or blood cavities. Both of these are very rare. A soft carcinoma grows rapidly, and the whole gland soon becomes fixed, so that a soft prostate which is fixed is highly suspicious of carcinoma.

The diagnosis between carcinoma and ehronic prostatitis may be extremely difficult. In both cases the gland is hard and irregular, and is approximately normal in size. The points in favour of prostatitis are, the preservation of the median furrow, the continued mobility of the gland, and the absence of gross extension outside the gland. An inflammatory prostate may show a slight degree of induration parallel to and below the seminal vesicles, but this is insufficient to render the gland fixed, and is never as marked as in malignant disease.

Little help is obtained by cystoscopy or urethroscopy. The bladder wall is usually trabeculated and hypertrophied, but the intravesical projection of a malignant prostate is generally insignificant. When present, it surrounds the internal meatus like a collar, and deep V-shaped depressions between the prostatic lobes are absent. On urethroscopy, the posterior urethra is found to be narrowed, especially above the verumontanum, and its surface may be irregularly nodular. The wide scabbard-shaped urethra of benign enlargement is not found.

The diagnosis of bone metastases is made by an X-ray examination. The first film should cover the whole of the lumbar spine, the bony pelvis and the upper thirds of the femora. If it does not reveal any abnormality, there is no necessity in going further, as the earliest bone metastases always take place in this area. If evidence of metastases is found, it is as well to take a film of the thorax and upper spine.

The bones may show patches of irregular thickening due to superficial growth of osseous tissue. If the original layer of superficial compact tissue remains, they may show a double contour, but as the malignant deposits increase the compact layer tends to be absorbed, and the double contour is no longer visible.

The cancellous tissue of the bone is profoundly altered. Its normal architecture is lost, and it looks as if it were composed of cotton wool. When changes of this nature are combined with a double contour, the diagnosis is beyond doubt. These changes stop abruptly at the joints. The intervertebral discs are never involved, and the same applies to large joints, such as the hip. The articular cartilage appears to be able to resist invasion. 
In other cases there is marked decalcification, with the result that large portions of the bone do not show up on the film. I have seen cases where one innominate bone was represented only by the iliac crest and the tuberosity of the ischium. In such cases the head of the femur appears to be completely unsupported. When changes of this type are found, one usually notices that other portions of the skeleton show thickening and sclerosis. This combination of osteoplastic and osteoclastic changes is highly suggestive of carcinoma.

These changes are occasionally diagnosed as Paget's disease of bone, and in many instances the resemblance is very close. The chief points of difference are-in Paget's disease the bones first affected are usually the skull, the dorsal spine, the pelvis, femur or tibia. In prostatic carcinoma the lumbar spine, pelvis and femur are always first affected, while changes in the skull and tibia are both late and relatively uncommon. In Paget's disease there is marked bending of the long bones, while in carcinoma they do not bend, but break. Paget's disease is much more chronic than carcinoma, and although the bones are thickened, irregular superficial nodules are absent.

Treatment. Carcinoma of the prostate commences insidiously and its earliest symptoms are due to obstruction. By then the growth has usually advanced beyond the capsule of the gland, so that one rarely meets with it in the operative stage. The result is that radical treatment has little scope, as in most instances the growth is inoperable when the patient is first seen. I have never succeeded in curing a patient in whom a positive diagnosis could be made on rectal examination.

In the rare cases where no spread beyond the limits of the gland, and no evidence of metastases have been found, one may try Young's radical operation. This consists in perineal excision of the prostate enclosed in its sheath of the pelvic fascia, together with the seminal vesicles and the base of the bladder. I have performed this operation on several occasions, but in every instance the patient died from distal metastases one to three years after the operation.

Interstitial radiation has also proved unsatisfactory in my hands. The method I employed was to open the bladder and insert needles containing I mgm. of radium element through the bladder base into the growth. These needles were inserted round the internal meatus, and also into the indurated tissue surrounding the seminal vesicles, which can easily be felt under the base of the bladder. A fortnight later, the patient was again anæsthetised, and the prostate exposed from the perineum. Similar needles were inserted into the lateral and posterior lobes of the gland. As a rule from 16 to 20 needles were inserted from above, and 6 to 8 from below. They were left in position for four to six days. In every instance the patient died from his carcinoma, the survival time being one to three years. High-voltage $\mathrm{X}$-ray treatment has also proved unavailing, the longest survival in my cases being two years.

There is no doubt in my mind that patients live longer after a permanent cystotomy than after any other operation. The disadvantages of such an operation are that unless the patient takes care to change his tube and wash out his bladder every day it will invariably become grossly infected, unless the tube fits accurately there is some leakage of urine and the patient's clothing always smells, and the tube is a constant reminder that he is suffering from an incurable disease. At the same time I have had many patients who have worn a suprapubic apparatus without much discomfort for four or five years. (The longest survival was $6 \frac{1}{4}$ years.)

Re-establishment of normal micturition can in many cases be brought about by per-urethral resection of the prostate. The obstructing portions of the gland are cut away by an endothermy loop through a McCarthy resectoscope, or some similar type of instrument. This operation does not aim at eradicating the whole of the carcinoma, but sufficient tissue is cut away to enable the patient to empty his bladder easily. Of course, partial operations always tend to accelerate the spread of any cancer, and shorten the patient's life. Against this should be placed the fact that he can live a practically normal life, and the knowledge that he is suffering from cancer may be hidden from him. Per-urethral resection cannot be carried out in every case. The urethra may be so much narrowed by the growth that it is impossible to introduce the instrument, and if it cannot be dilated easily the operation should be abandoned. Again, if the patient is in a state of complete retention before the operation, it may be followed by 
incontinence. This only occurs when the compressor urethræ is infiltrated with growth, and cannot act as a sphincter. In these cases the obstruction is at the level of the internal meatus. If the proximal portion of the urethra is cut away, the patient depends on the compressor, which is already damaged. One can usually recognise infiltration of the compressor on rectal examination, when the induration spreads downwards below the apex of the prostate and involves the region of the membranous urethra.

Enucleation is rarely possible in this type of carcinoma, as the growth rapidly involves the sheath of the gland, and obliterates the plane of cleavage.

\section{Carcinoma arising in an adenomatous prostate.}

In this condition two types of cases are encountered. In the first no suspicion of carcinoma is aroused either before or during operation, but an area of carcinoma is found on microscopic examination. In the second, the malignant disease can be diagnosed clinically.

I have already stated that malignant changes have been found in from 4 to 20 per cent. of apparently benign glands. The carcinoma does not give rise to signs or symptoms, and enucleation of the prostate is easy, but a small carcinomatous area is found on microscopic examination. This is, in my opinion, the only form of prostatic carcinoma that is curable. These cases usually do well, a recurrence is only found in about 5 per cent. of them (ThomsonWalker). It usually arises in the prostatic bed, and may cause a return of the obstructive symptoms. If it does, a fresh passage can be made by means of the resectoscope.

The second type of case is a more advanced stage of that just described. The carcinoma can be diagnosed, or at least suspected, on rectal examination. One feels a considerably enlarged gland. The lateral lobes are well defined, and are separated from each other by a well marked median furrow. At one spot, usually at the periphery of one lateral lobe, there is a hard, rather prominent mass, which is often crescentic in outline. This side of the prostate is less movable than the other, and there may be a band of induration spreading upwards and outwards below the corresponding seminal vesicle. The remainder of the gland is usually soft, but it may be a little harder than an ordinary adenomatous prostate.

The greater part of the prostate can be enucleated fairly easily, but in the neighbourhood of the hard nodule it is adherent, and invariably a small portion is left behind. After removal, the prostatic bed feels thickened and hard over this area. If the condition is more advanced enucleation may be extremely difficult, or even impossible, and portions of the prostate have to be cut out with a scissors. In these cases there is a tendency for the seminal vesicles to come away with the gland. If this happens, a very large cavity is left, but hæmorrhage can always be controlled by light packing.

The results of this type of operation are variable. In some cases the patient remains free from symptoms for several years, and ultimately dies of metastatic deposits. In most instances, however, the obstruction rapidly recurs. Occasionally this may happen before the suprapubic wound closes, leaving a permanent fistula. If the recurrence is noticed before the urethra is completely blocked, and it is still possible to pass instruments, the obstructing tissue, which is usually situated at the internal meatus, may be cut away through an operating urethroscope. When the obstruction is complete, and instrumentation is impossible, it is better to establish a permanent suprapubic fistula.

On account of the risk of a recurrence of the obstruction, I think it better to perform a per-urethral resection of the prostate or a simple cystotomy than to enucleate the gland. 